\title{
Pseudo-Orbital SO(6) Symmetry for pf-Shell Nuclei
}

\author{
J. P. Valencia and H.C. Wu \\ Instituto de Fisica, Universidad de Antioquia, Medellin, Colombia
}

Received on 6 September, 2006

\begin{abstract}
This work studies the pseudo-orbital SO(6) symmetry for the first half of the $2 \mathrm{p}_{\frac{1}{2}}-2 \mathrm{p}_{\frac{3}{2}}-1 \mathrm{f}_{\frac{5}{2}}$ shell (denoted as the pf-shell). The spectra and beta decay are calculated for nuclei $\mathrm{Ni}, \mathrm{Cu}$ and $\mathrm{Zn}$ with $\mathrm{A}=58$, and the comparison to experiment prefers the $\widetilde{\mathrm{SO}}(6)$ limit to the $\widetilde{\mathrm{SU}}(3)$ one. In the calculation of beta decay two types of transformations are employed and the results seem confirm the adequacy of p-helicity transformation.
\end{abstract}

Keywords: Nuclear structure; Pseudo-orbital SO(6) symmetry; Beta decay; pf-shell nuclei

\section{INTRODUCTION}

The concept of pseudo-spin and pseudo-orbit was introduced in $[1,2]$ to account for symmetries for nuclei with large 1-s coupling. Later it was shown that pseudo-spin has a root in the relativistic mean field theory [3], therefore, the study of pseudo symmetries can be put in a new perspective and thus appears more attractive. Pseudo-spin and pseudo-orbits have been employed in several symmetry models. The most successful example is the pseudo-SU(3) (denoted as $\widetilde{\mathrm{SU}}(3)$ ) model (see a recent article [4] and references therein). The Fermion Dynamical Symmetry Model (FDSM) [5] also uses pseudo-spin and pseudo-orbits, however, in the FDSM either pseudo-spin or pseudo-orbits are deactivated. The $\widetilde{\mathrm{SU}}(4)$ model $[6,7]$ combines pseudo-spin and isospin. The exploration of the $\widetilde{\mathrm{SU}}(4)$ symmetry is pursued in two ways. The first method analyzes the $\widetilde{\mathrm{SU}}(4)$ content of wave functions obtained from a shell model calculation, and the method can be referred to as "microscopic analysis". The second (referred to as "application"), by assuming the $\widetilde{\mathrm{SU}}(4)$ symmetry, calculates energies and transitions with group theoretical method and compares the results to experiments. The two methods check the validity of the $\widetilde{\mathrm{SU}}(4)$ symmetry in different but complimentary ways. Ref. [6] followed the first line and it gave mixed results, whereas ref. [7] used both methods and concluded the approximate validity of the $\widetilde{\mathrm{SU}}(4)$ classification scheme. To be more specific, ref. [7] analyzed microscopically the pf-shell nuclei ${ }^{58} \mathrm{Ni},{ }^{60} \mathrm{Ni}$ and ${ }^{60} \mathrm{Zn}$, applied the model in the study of beta decay, and the $\widetilde{\mathrm{SU}}(4)$ symmetry was confirmed by both methods.

In this work we intend to explore the $\widetilde{\mathrm{SU}}(4)$ symmetry along the line of application by employing new symmetry elements. In the application of the $\widetilde{\mathrm{SU}}(4)$ model in ref. [7] the pseudo-orbital symmetry was taken as $\widetilde{\mathrm{SU}}(3)$. However, we argue that the pseudo-orbits in this shell are $\tilde{s}$ and $\tilde{d}$, therefore the maximal pseudo-orbital symmetry should be $\widetilde{\mathrm{SU}}(6)$. As is well known that the $\widetilde{\mathrm{SU}}(6)$ group has three subgroups (hereafter denoted as limits), i.e. the $\widetilde{\mathrm{SU}}(3), \widetilde{\mathrm{SO}}(6)$ and $\widetilde{\mathrm{SU}}(5)$, respectively, and the limits of $\widetilde{\mathrm{SO}}(6)$ and $\widetilde{\mathrm{SU}}(5)$ were never studied before. While the group structure of the three limits is the same as that of the Interacting Boson Model (IBM) [8], the physical contents are quite different: IBM deals with boson systems, whereas our model works in a fermion space and the relevant irreducible representations (irreps) are different. It is the goal of this work to study the $\widetilde{\mathrm{SO}}(6)$ pseudoorbital limit for the pf-shell nuclei, taking the $\mathrm{A}=58$ nuclei as an initial step. The spectrum of the $\widetilde{\mathrm{SO}}(6)$ limit is examined in Section 2 and $\beta$-decay calculated in Section 3. Conclusion and discussion are given in Section 4.

\section{THE $\widetilde{\mathrm{SO}}(6)$ SPECTRUM}

It is reasonable to expect that there exist substantial differences between the $\widetilde{\mathrm{SO}}(6)$ and $\widetilde{\mathrm{SU}}(3)$ limits. First we examine a special case that is the pseudo-orbital wave functions of the ground state $(\tilde{L}=0)$ for $\mathrm{A}=58$ nuclei. The pseudo-orbital wave functions are the same as the normal-orbital ones in the ds shell (ref. [9]) except that the normal orbits d and s are replaced by pseudo-orbits $\tilde{d}$ and $\tilde{s}$. The wave functions in the two limits are:

$$
\begin{aligned}
\widetilde{\mathrm{SU}}(3): \mid[2](40) S> & =\sqrt{\frac{5}{9}}\left|\left(\tilde{s}^{2}\right) S>+\sqrt{\frac{4}{9}}\right|\left(\tilde{d}^{2}\right) S>, \\
\widetilde{\mathrm{SO}}(6): \mid[2](200)(00) S> & =\sqrt{\frac{5}{6}}\left|\left(\tilde{s}^{2}\right) S>-\sqrt{\frac{1}{6}}\right|\left(\tilde{d}^{2}\right) S>.
\end{aligned}
$$

A big difference exists between the wave functions of the two $\tilde{d}^{2}$, are mixed with almost equal probability, in the $\widetilde{\mathrm{SO}}(6)$ limit limits: while in the $\widetilde{\mathrm{SU}}(3)$ limit the two configurations, $\tilde{s}^{2}$ and 
the $\tilde{s}^{2}$ configuration is dominant ( $83 \%$ of probability). This difference can be expected to produce significant differences in spectra and transitions.

We first discuss the spectra. A general energy formula in the $\widetilde{\mathrm{SO}}(6)$ limit can be written as follows,

$$
\begin{aligned}
E= & a_{0}<\hat{n}>+a_{1}<\tilde{M}>+a_{T} T(T+1) \\
& +a_{L} \tilde{L}(\tilde{L}+1)+a_{J}\langle\tilde{L} \cdot \tilde{S}\rangle \\
& +a_{5}<C_{\mathrm{SO} 5}>+a_{6}<C_{\mathrm{SO} 6}>
\end{aligned}
$$

where $\hat{n}$ and $\tilde{M}$ are the Casimir operators of $\mathrm{U}(24)$ and $\widetilde{\mathrm{SU}}(6)$ groups; $\tilde{S}, T, \tilde{L}$ and $J$ the pseudo-spin, isospin, pseudo-orbital and total angular momenta, and $C_{\mathrm{SO} 6}$ and $C_{\mathrm{SO} 5}$ the Casimir operators of the groups $\widetilde{\mathrm{SO}}(6)$ and $\widetilde{\mathrm{SO}}(5)$, respectively. There is no $\tilde{S}$ term in eq. (2), since it can be taken as complimentary to $T$. Concerning the calculation in the pseudo-orbital space, the group theoretical method of IBM [8] can be used.

As an initial step, in this work we study the $\widetilde{\mathrm{SO}}(6)$ limit for the three nuclei with $\mathrm{A}=58$. The spectra of ${ }^{58} \mathrm{Cu}$ consists of two $(\tilde{S}, T)$ pairs, $(1,0)$ and $(0,1)$, while the spectra of ${ }^{58} \mathrm{Ni}$ and ${ }^{58} \mathrm{Zn}$ have only one $(\tilde{S}, T)$ pair, $(0,1)$. There is only one $\widetilde{\mathrm{SU}}(6)$ irrep involved and the $\widetilde{\mathrm{SU}}(6) \supset \widetilde{\mathrm{SO}}(6) \supset \widetilde{\mathrm{SO}}(5) \supset \widetilde{\mathrm{SO}}(3)$ branching writes as

$$
[2] \supset(200)+(000) \supset(20)+(10)+(00)+(00) \supset(\tilde{L}=) 0,2,4,2,0 \text {. }
$$

The branching is the same for $(\tilde{S}, T)=(0,1)$ and $(1,0)$. Note that the tilde signs of all the pseudo irreps are neglected for simplicity. The calculated and experimental levels are given in Figure 1. The theoretical energies contain pure nuclear interaction, and the experimental ones are taken from $[10,11]$ and processed such that the Coulomb energy is subtracted and the result is normalized to the ground state energy of ${ }^{58} \mathrm{Cu}$. For every nucleus the Coulomb energy is taken equal for all the levels. The value of $\tilde{L}$ is given at the right hand side of each calculated level in order to identify pseudo L-S partners.

Since all the levels of the three nuclei belong to the same $\widetilde{\mathrm{SU}}(6)$ irrep, the first two terms in eq. (2) can be ignored (note that these terms are indispensable for $\mathrm{A}=59$ and 60 nuclei). The rest five parameters can be derived from fitting the sixteen experimental levels and the results are:

$$
\begin{gathered}
a_{T}=0.150, a_{L}=-0.008, a_{J}=-0.220 ; \\
a_{5}=0.280, a_{6}=-0.243
\end{gathered}
$$

(all the parameters are in $\mathrm{MeV}$ ).

From a comparison between experiment and calculation, the following observations can be made. First, all the $T=1$ states of the three nuclei are reproduced reasonably well except the $1_{1}^{+}$state of ${ }^{58} \mathrm{Ni}$, which may belong to a higher $\widetilde{\mathrm{SU}}(6)$ irrep. There exist two almost identical level series, i.e. $0_{1}^{+}-2_{1}^{+}-4_{1}^{+}$, in the spectra of ${ }^{58} \mathrm{Ni}$ and ${ }^{58} \mathrm{Cu}$, which are the components of an isospin triplet (the $2_{1}^{+}-4_{1}^{+}$states of ${ }^{58} \mathrm{Zn}$ are currently not available). And the energy differences among the $0_{1}^{+}$states of ${ }^{58} \mathrm{Ni},{ }^{58} \mathrm{Cu}$ and ${ }^{58} \mathrm{Zn}$ are less than 100 $\mathrm{KeV}$ while the total nuclear energies are around $634 \mathrm{MeV}$. All these are consequences of the $\mathrm{SU}(2)_{\mathrm{T}}$ symmetry. The ratio $\rho=\left(E_{4_{1}^{+}}-E_{0_{1}^{+}}\right) /\left(E_{2_{1}^{+}}-E_{0_{1}^{+}}\right)$is calculated as $\approx 2.4$ for $\mathrm{Ni}$ and $\mathrm{Cu}$, that is much closer to its experiment value $(\approx 1.7)$ than the $\widetilde{\mathrm{SU}}(3)$ prediction $(\approx 3.3$, ref. [8]). Second, the $T=0$ states of ${ }^{58} \mathrm{Cu}$ provide an opportunity to verify the validity of $\widetilde{\mathrm{SU}}(4) \otimes \widetilde{\mathrm{SU}}(6)$ symmetry, since this symmetry unifies the states of $T=0$ and 1 . The four lowest states, $1_{1}^{+}, 3_{1}^{+}, 2_{1}^{+}$and $1_{2}^{+}$, are reproduced fairly well except that the order of the two states, $2_{1}^{+}$and $1_{2}^{+}$, is reversed. The three states with higher energies, i.e. $4_{1}^{+}, 3_{1}^{+}, 5_{1}^{+}$, are also predicted by the theory, however there is a considerable discrepancy between the experimental and theoretical energies. The $\widetilde{\mathrm{SO}}(6)$ limit seems also preferred concerning the $T=0$ states of ${ }^{58} \mathrm{Cu}$, since the prediction of $\tilde{L}=4$ state in the $\widetilde{\mathrm{SU}}(3)$ limit would be much higher (than that of $\widetilde{\mathrm{SO}}(6)$ ), and consequently the discrepancy in energy between theory and experiment for the states of $4_{1}^{+}, 3_{2}^{+}, 5_{1}^{+}$could be much larger.

For the nuclei with $\mathrm{A}=59$ and 60 , the $\widetilde{\mathrm{SU}}(6) \supset \widetilde{\mathrm{SO}}(6) \supset$ $\widetilde{\mathrm{SO}}(5) \supset \widetilde{\mathrm{SO}}(3)$ branching can be derived by using the method of ref. [12]. A study on these nuclei is underway.

\section{III. $\beta$ DECAY AND THE P-HELICITY TRANSFORMATION}

Beta decay provides a serious check on wave functions. The Gamow-Teller operator in the pseudo formalism can be written as,

$$
\begin{aligned}
\hat{M}_{\mathrm{GT}}= & \sum_{q} \vec{\tau}(q) \tilde{\tilde{\sigma}}(q)=-\frac{1}{3} \sum_{q} \vec{\tau}(q) \vec{\sigma}(q)-\frac{2 \sqrt{10}}{3} \sum_{q} \vec{\tau}(q)\left[C_{\lambda}^{(2)}(q) \otimes \vec{\sigma}(q)\right]^{(1)} \\
& \left(\equiv \hat{M}_{\mathrm{GT}}^{S}+\hat{M}_{\mathrm{GT}}^{L S}\right),
\end{aligned}
$$




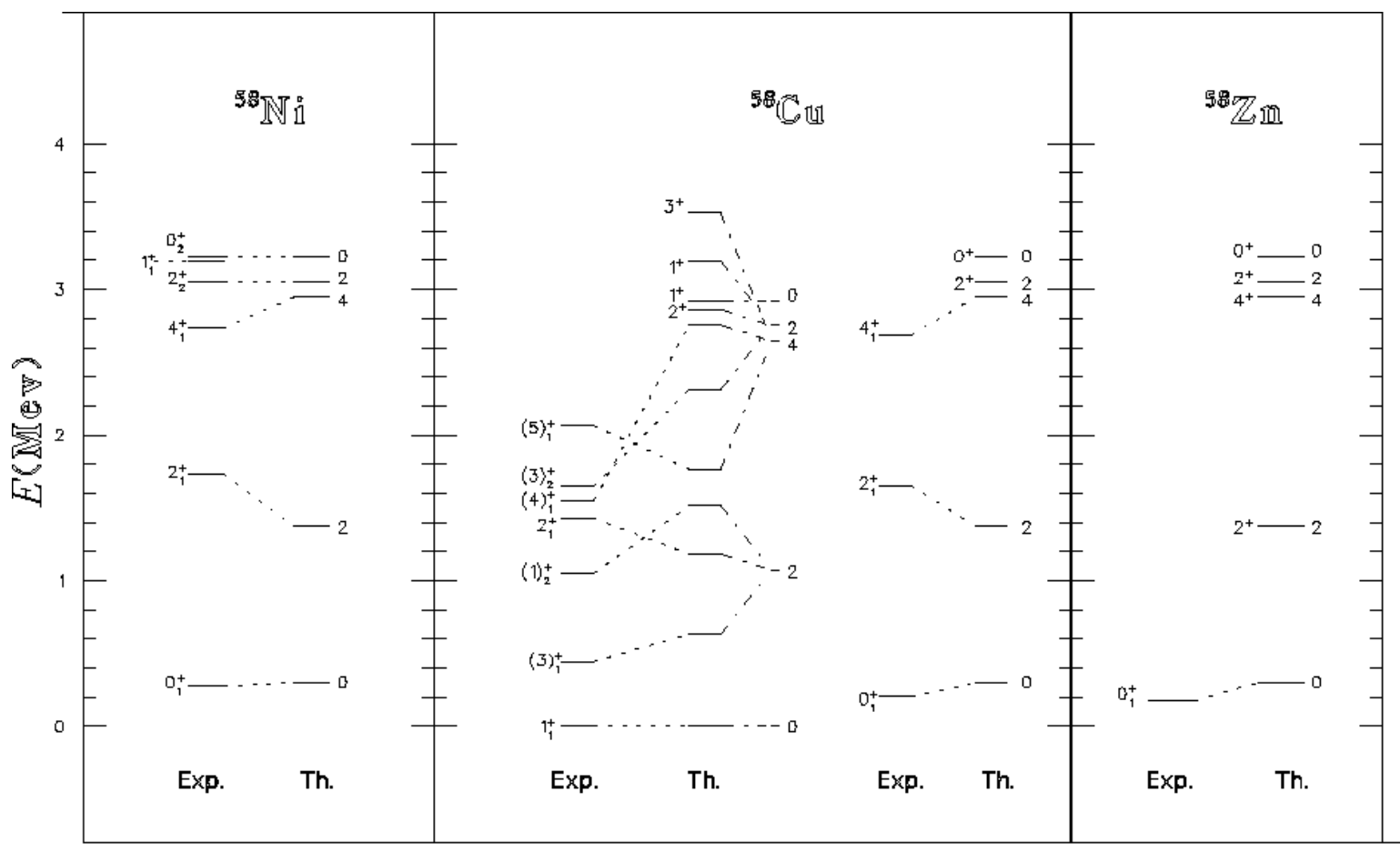
$(\tilde{S}, T)=$
$(0,1)$
$(1,0)$
$(0,1)$
$(0,1)$

FIG. 1: The $\widetilde{\mathrm{SO}}(6)$ spectra of the Ni-Cu-Zn nuclei with $\mathrm{A}=58$. The theoretical energies are pure nuclear interactions. The experimental levels are taken from ref. $[10,11]$ and they are processed such that the Coulomb energy is subtracted and the result is normalized to the ground state energy of ${ }^{58} \mathrm{Cu}$. $(\tilde{S}, T)$ is the (pseudo-spin)-isospin quantum number pair. $\tilde{L}$ at the r.h.s. of each calculated level is the pseudo-orbital angular momentum. $J^{\pi}$, the total angular momentum and parity, is given at the 1.h.s. for each experimental level and also for the calculated levels which have no experimental counterpart.

where $\mathrm{q}$ is particle index, and $C_{\lambda}^{(2)}(q)$ is a rank-2 operator whose concrete form depends on the normal $\rightarrow$ pseudo transformation. When the r-helicity transformation $(\lambda=r)[13]$ is employed, eq. (5) is the same as eq. (6) in ref. [7]. The two terms in the above equation are called the spin term $\left(\hat{M}_{\mathrm{GT}}^{S}\right)$ and the spin-orbit coupling term $\left(\hat{M}_{\mathrm{GT}}^{L S}\right)$, respectively.

In ref. [7] the r-helicity transformation is used. However, it was pointed out recently in ref. [14] that the r-helicity transformation may be only a good approximation for heavy nuclei, and the correct transformation is that of p-helicity $(\lambda=p)$ $[15,16]$. In this work we calculate the Gamow-Teller matrix elements by using the p-helicity transformation and compare the result to the r-helicity one. The zero-th component of the $C_{p}^{(2)}$ operator writes as follows,

$$
\sqrt{\frac{3}{2}} \frac{(\vec{p} \times \vec{p})_{0}^{(2)}}{p^{2}}=-\frac{3}{2} \frac{\hbar^{2} \nabla_{z}^{2}}{p^{2}}-\frac{1}{2},
$$

in which the denominator $p^{2}$ takes a numerical value for eigenstate as is given by the Schrödinger equation:

$$
p^{2}=2 \mu(E-V(r))
$$

where $\mu$ is the nucleon mass, and $E$ and $V(r)$ are the singleparticle total and potential energies, respectively. In this
TABLE I: Reduced matrix elements of the quadrupole operator for $\mathrm{A}=58$ nuclei

\begin{tabular}{c|ccc}
\hline \hline Helicity & $\left.\left\langle 0|| C^{(2)} \| 2\right\rangle<2\left\|C^{(2)}\right\| 0\right\rangle<2\left\|C^{(2)}\right\| 2>$ \\
\hline $\mathrm{r}$ & 0.775 & 0.775 & -1.195 \\
\hline $\mathrm{p}$ & -0.088 & 0.055 & 1.842 \\
\hline
\end{tabular}

work we restrict ourselves to a schematic calculation on $C_{p}^{(2)}$, and thus we use the harmonic oscillator wave functions for pseudo-orbits with parameters being taken following ref. [17].

In Table 1 the reduced matrix elements are listed for the $r$ and p-helicity cases, respectively. There exists a drastic difference between the two helicity cases: while the reduced matrix elements in p-helicity are close to zero for the case of unequal initial and final angular momenta, their counterparts in r-helicity are of the order of 1 . As a consequence, the calculated beta transitions for the two helicity cases will be substantially different.

The Gamow-Teller transition rates are calculated by using the group theoretical method, and the relevant formulas are listed in Appendix. In Table 2, four Gamow-Teller transitions are listed, and a Fermi transition is given for comparison. In 
TABLE II: Beta decay of Ni-Cu-Zn nuclei with $\mathrm{A}=58$

\begin{tabular}{|c|c|c|c|c|c|c|c|c|c|}
\hline No. & Transition & $J_{i}^{\pi} \quad T_{i}$ & $E_{i}$ & $J_{f}^{\pi} \quad T_{f}$ & $E_{f}$ & Limit-helicity & Contributing Term & $\log (\mathrm{ft})_{\text {th }}$ & $\log (\mathrm{ft})_{\exp }$ \\
\hline 1 & ${ }^{58} \mathrm{Cu} \rightarrow{ }^{58} \mathrm{Ni}$ & $1_{1}^{+} \quad 00$ & & $0_{1}^{+} \quad 1$ & 0.0 & & $\hat{M}_{\mathrm{GT}}^{S}$ & 4.51 & 4.87 \\
\hline \multirow[t]{4}{*}{2} & & $1_{1}^{+} \quad 0$ & & $22_{1}^{+} 1$ & 1.454 & $\widetilde{\mathrm{SO}}(6)-\mathrm{r}$ & $\hat{M}_{\mathrm{GT}}^{L S}$ & $4.05 \sim 4.40$ & 6.2 \\
\hline & & & & & & $\widetilde{\mathrm{SU}}(3)-\mathrm{r}$ & $\hat{M}_{\mathrm{GT}}^{L S}$ & $3.95 \sim 4.56$ & \\
\hline & & & & & & $\widetilde{\mathrm{SO}}(6)-\mathrm{p}$ & $\hat{M}_{\mathrm{GT}}^{L S}$ & $6.84 \sim 6.27$ & \\
\hline & & & & & & $\widetilde{\mathrm{SU}}(3)-\mathrm{p}$ & $\hat{M}_{\mathrm{GT}}^{L S}$ & $4.81 \sim 4.75$ & \\
\hline 3 & ${ }^{58} \mathrm{Zn} \rightarrow{ }^{58} \mathrm{Cu}$ & $0_{1}^{+} 11$ & & $1_{1}^{+} 0$ & 0.0 & & $\hat{M}_{\mathrm{GT}}^{S}$ & 4.03 & 4.3 \\
\hline \multirow[t]{4}{*}{4} & & $0_{1}^{+} \quad 1 \mathrm{C}$ & & $1_{2}^{+} 0$ & 1.052 & $\widetilde{\mathrm{SO}}(6)-\mathrm{r}$ & $\hat{M}_{\mathrm{GT}}^{L S}$ & $3.57 \sim 3.93$ & 4.1 \\
\hline & & & & & & $\widetilde{\mathrm{SU}}(3)-\mathrm{r}$ & $\hat{M}_{\mathrm{GT}}^{L S}$ & $3.48 \sim 4.08$ & \\
\hline & & & & & & $\widetilde{\mathrm{SO}}(6)-\mathrm{p}$ & $\hat{M}_{\mathrm{GT}}^{L S}$ & $6.37 \sim 5.79$ & \\
\hline & & & & & & $\widetilde{\mathrm{SU}}(3)-p$ & $\hat{M}_{\mathrm{GT}}^{L S}$ & $4.34 \sim 4.28$ & \\
\hline 5 & & $0_{1}^{+} \quad 1 \quad 0$ & & $0_{1}^{+} 1$ & 0.203 & & $\hat{M}_{\mathrm{F}}$ & 3.46 & 3.48 \\
\hline
\end{tabular}

the first column of the table a number is given to represent each transition. $J_{i}^{\pi}, T_{i}, E_{i}$ and $J_{f}^{\pi}, T_{f}, E_{f}$ are the (total angular momentum)-parity, isospin and experimental energy for the initial and final states, respectively. $\hat{M}_{\mathrm{GT}}^{S}, \hat{M}_{\mathrm{GT}}^{L S}$ are the spin term and spin-orbit coupling term of the Gamow-Teller operator, and $\hat{M}_{\mathrm{F}}$ is the Fermi transition operator, respectively. The $\log (f t)_{\exp }$ values are taken from refs $[18,19]$. In our calculation a hindrance factor of 0.53 is used following ref. [20].

A prominent feature of the Gamow-Teller matrix elements (for $\mathrm{A}=58$ ) is that, for each transition, the contribution of either $\hat{M}_{\mathrm{GT}}^{S}$ or $\hat{M}_{\mathrm{GT}}^{L S}$ is zero. Transitions 1 and 3 are driven solely by the term $\hat{M}_{\mathrm{GT}}^{S}$, thus they are independent of the pseudo-orbital wave functions, i.e. the initial and final pseudo-orbital wave functions are identical (the $\widetilde{\mathrm{SO}}(6)$ state $\mid[2](200)(00) 0>)$. The calculated decay rates of these two transitions are close to experiments. Transitions 2 and 4 are produced by the spin-orbit coupling term, $\hat{M}_{\mathrm{GT}}^{L S}$, thus the transition rate depends on the pseudo-orbital wave functions. In the $\widetilde{\mathrm{SO}}(6)$ limit, the two transitions have the same initial pseudo-orbital state $(\mid[2](200)(00) 0>$ ) and the same final state $(\mid[2](200)(10) 2>)$, however, a difference in $\tilde{S}, T$ and $J$ makes difference in decay rates. The decay rates are calculated for the p-helicity as well as r-helicity cases. For a comparison, corresponding results are also given for the $\widetilde{\mathrm{SU}}(3)$ limit. In Table 2 the symbol $\widetilde{\mathrm{SO}}(6)-\mathrm{r}$ means that the pseudoorbital wave function is in the $\widetilde{\mathrm{SO}}(6)$ limit and the calculation is done for the r-helicity case, etc. Two boundary values are given for each case corresponding to the two choices of phase in the coefficients of fractional parentage (cfp) of $\widetilde{\mathrm{SO}}(6)$ [21] and $\widetilde{\mathrm{SU}}(3)$ [7], respectively. One notices that, for the r-helicity case, the results of the two limits, $\widetilde{\mathrm{SO}}(6)$ and $\widetilde{\mathrm{SU}}(3)$, are very close, despite that the wave functions in the two limits are quite different. It is not yet known if this near equality of decay rates of the two limits for the r-helicity case is a common phenomenon or just a coincidence for the case of $\mathrm{A}=58$, thus further study is needed. In the p-helicity case the tran- sition rates of the $\widetilde{\mathrm{SO}}(6)$ and $\widetilde{\mathrm{SU}}(3)$ limits are very different. While the $\widetilde{\mathrm{SO}}(6)$ limit explains well transition 2 , the $\widetilde{\mathrm{SU}}(3)$ limit gives a better result for transition 4 . From the spectrum (Fig. 1) one may assume that the $1_{2}^{+}$state $(T=0)$ of ${ }^{58} \mathrm{Cu}$ has a considerable mixing with other states since the order of the states $1_{2}^{+}$and $2_{1}^{+}$is reversed and there are many (uncertain) states nearby [10] which could facilitate a mixing. Should this assumption be correct, the calculation of transition 4 would be less reliable. From transition 2, one can assert that the phelicity transformation is more proper for the nuclei studied, and the $\widetilde{\mathrm{SO}}(6)$ limit is preferred to the $\widetilde{\mathrm{SU}}(3)$. However, this statement needs to be checked by more studies, in particular by a consistent calculation with mixed symmetry on both the energy and beta decay concerning the $1_{2}^{+}$state of ${ }^{58} \mathrm{Cu}$.

Finally we note that the four Gamow-Teller transitions listed in Table 2 are among three nuclei, and they are calculated in an unified way with the same set of parameters. The transition rates are reproduced qualitatively well, which is an encouraging sign for the $\widetilde{\mathrm{SU}}(4) \otimes \widetilde{\mathrm{SU}}(6)$ symmetry.

\section{CONCLUSIONS AND DISCUSSION}

This work discusses the pseudo-orbital $\mathrm{SO}(6)$ limit that was not studied before. As an initial step the spectra and Gamow-Teller transitions of the nuclei ${ }^{58} \mathrm{Ni},{ }^{58} \mathrm{Cu}$ and ${ }^{58} \mathrm{Zn}$ are analyzed in the $\widetilde{\mathrm{SO}}(6)$ limit. The spectra of the three nuclei are described by a single formula. At first glance, it seems not appealing by using five parameters to fit sixteen levels. However, considering that we are dealing with three nuclei which are of different type (even-even and odd-odd), the agreement should be considered as reasonable. The ratio of $\left(E_{4_{1}^{+}}-E_{0_{1}^{+}}\right) /\left(E_{2_{1}^{+}}-E_{0_{1}^{+}}\right)$is better predicted by the $\widetilde{\mathrm{SO}}(6)$ limit than the $\widetilde{\mathrm{SU}}(3)$ one. Moreover the lowest $T=0$ states of ${ }^{58} \mathrm{Cu}$ are reproduced fairly well by the $\widetilde{\mathrm{SO}}(6)$ limit. All these seem prefer the $\widetilde{\mathrm{SO}}(6)$ limit to the $\widetilde{\mathrm{SU}}(3)$ one. We note that in 
this work the $\widetilde{\mathrm{SO}}(6)$ limit was chosen on an ad hoc basis, and the study of the $\widetilde{S U}(5)$ limit is equally important. In particular, a comparison among the three limits may reveal essential differences among them. Probably, a mixing of limits may be needed to obtain quantitative agreement with experiment.

The Gamow-Teller transitions are calculated in the phelicity as well as the r-helicity cases. One finds that there exists a drastic difference between these two helicities for the transitions that are driven by the term $\hat{M}_{\mathrm{GT}}^{L S}$. For transitions 2 in Table 2, the p-helicity calculation gives better results than the r-helicity one, which seems confirm that the appropriate transformation is the p-helicity one. Although the calculation in this work is only schematic, the qualitative feature of the matrix elements of $C_{p}^{(2)}$ (as is shown in Table 1) will probably remain in a more realistic calculation, since the operator $\nabla_{z}^{2}$ has a small expectation value on all states. However, a more realistic pseudo-orbital wave function may change the matrix elements of $C_{p}^{(2)}$ quantitatively, and in turn the transition rates could be changed considerably. The study of the $\widetilde{\mathrm{SO}}(6)$ limit for $\mathrm{A}=58$ nuclei is only an initial step, it would be interesting to see if the conclusion of this work will persist for nuclei with $A=59$ and 60 , and the study is currently underway.

\section{Appendix}

With an expansion in the one-body cfp of the relevant group chain, the matrix elements of the Gamow-Teller operator are derived for a general case (which applies to all the three pseudo-orbital limits). The results are,

$$
\begin{aligned}
\left\langle\hat{M}_{\mathrm{GT}}\right\rangle= & \sqrt{\frac{2 J_{f}+1}{\left(2 T^{\prime}+1\right)}}\left\langle T M_{T} 1 v \mid T^{\prime} M_{T^{\prime}}\right\rangle\left\langle\left[f^{\prime}\right] \nVdash \tau \sigma \nVdash[f]\right\rangle \\
& \left(\frac{(-1)^{J_{f}+S+L}}{3}\left\{\begin{array}{ccc}
J_{i} & J_{f} & 1 \\
S^{\prime} & S & L^{\prime}
\end{array}\right\} \delta_{L^{\prime} L} \delta_{[f]\left[f^{\prime}\right]}-2 \sqrt{\frac{10}{3}}\left\{\begin{array}{ccc}
L^{\prime} & L & 2 \\
S^{\prime} & S & 1 \\
J_{f} & J_{i} & 1
\end{array}\right\} \mathbb{F}_{L}^{2}\right),
\end{aligned}
$$

where the (pseudo-spin)-isospin factor reads,

$$
\begin{aligned}
\left\langle\left[f^{\prime}\right] \nVdash \tau \sigma \nVdash[f]\right\rangle= & \left.\left.n \frac{N_{\left[f^{\prime \prime}\right]}}{\sqrt{\left.N_{[f]} N_{\left[f^{\prime}\right]}\right] \beta^{\prime \prime} S^{\prime \prime} T^{\prime \prime}}}\left\langle\left[f^{\prime \prime}\right] \beta^{\prime \prime} S^{\prime \prime} T^{\prime \prime} ;[1] \frac{1}{2} \frac{1}{2}\right|\right\}\left[f^{\prime}\right] \beta^{\prime} S^{\prime} T^{\prime}\right\rangle \\
& \left.\left.\left\langle\left[f^{\prime \prime}\right] \beta^{\prime \prime} S^{\prime \prime} T^{\prime \prime} ;[1] \frac{1}{2} \frac{1}{2}\right|\right\}[f] \beta S T\right\rangle(-1)^{S^{\prime \prime}+\frac{1}{2}+S^{\prime}+1} \sqrt{\left(2 S^{\prime}+1\right)(2 S+1)}\left\{\begin{array}{ccc}
S & S^{\prime} & 1 \\
\frac{1}{2} & \frac{1}{2} & S^{\prime \prime}
\end{array}\right\} \\
& (-1)^{T^{\prime \prime}+\frac{1}{2}+T^{\prime}+1} \sqrt{\left(2 T^{\prime}+1\right)(2 T+1)}\left\{\begin{array}{ccc}
T & T^{\prime} & 1 \\
\frac{1}{2} & \frac{1}{2} & T^{\prime \prime}
\end{array}\right\}\left\langle\frac{1}{2} \frac{1}{2} \nVdash \tau \sigma \nVdash \frac{1}{2} \frac{1}{2}\right\rangle,
\end{aligned}
$$

and the pseudo-orbital factor $\mathbb{F}_{L}^{(2)}$ is

$$
\begin{aligned}
& \left.\left.\left.\left.\left.\mathbb{F}_{L}^{k}=\sum_{\substack{\alpha^{\prime \prime} L^{\prime \prime} \\
l^{\prime} l}}\left\langle\widetilde{f^{\prime \prime}}\right] \alpha^{\prime \prime} L^{\prime \prime} ;[\tilde{1}] l \mid\right\}[\tilde{f}] \alpha L\right\rangle\left\langle\widetilde{f^{\prime \prime}}\right] \alpha^{\prime \prime} L^{\prime \prime} ; \tilde{1}\right] l^{\prime} \mid\right\}\left[\tilde{f}^{\prime}\right] \alpha^{\prime} L^{\prime}\right\rangle \\
& \quad(-1)^{L^{\prime \prime}+l+L^{\prime}+k} \sqrt{\left(2 L^{\prime}+1\right)(2 L+1)}\left\{\begin{array}{ccc}
L & L^{\prime} & k \\
l^{\prime} & l & L^{\prime \prime}
\end{array}\right\}\left\langle l^{\prime}\left\|C^{k}\right\| l\right\rangle \quad(k=0,2),
\end{aligned}
$$

where $N_{[f]}$ is the dimension of the symmetric group irrep [f], etc; $\left.\left.\left\langle\left[f^{\prime \prime}\right] \beta^{\prime \prime} S^{\prime \prime} T^{\prime \prime} ;[1] \frac{1}{2} \frac{1}{2}\right|\right\}[f] \beta S T\right\rangle \quad$ is the $\left.\left.\mathrm{SU}(4) \supset \mathrm{SU}(2)_{S} \otimes \mathrm{SU}(2)_{T} \operatorname{cfp}[2] ;\left\langle\left[f^{\prime \prime}\right] \alpha^{\prime \prime} L^{\prime \prime} ;[\tilde{1}] l\right|\right\}[\tilde{f}] \alpha L\right\rangle$ is the cfp of the relevant pseudo-orbital group chain, respectively. In the present work the cfp with $\alpha$ is that of the group chain $\mathrm{SU}(6) \supset \mathrm{SO}(6) \supset \mathrm{SO}(5) \supset \mathrm{SO}(3)$ where $\alpha$ is an abbreviation of the quantum numbers of the groups of $\mathrm{SO}(6)$ and $\mathrm{SO}(5)$. The quantities without prime, with single and double primes correspond to the initial, final and intermediate states, respectively. We note that all the tilde signs in Appendix means conjugation in representations.

\section{Acknowledgments}

The authors thank Colciencias and the University of Antioquia, Colombia for financial support. We are grateful to the 
Institute of Theoretical Physics, CAS, China for their hospitality during their visit. Interesting discussions with prof. E.G.
Zhao are greatly appreciated.
[1] A. Arima, M. Harvey, and K. Shimizu, Phys. Lett. B30, 517(1969)

[2] K.T. Hecht and A. Adler, Nucl. Phys. A137, 129(1969).

[3] J.N. Ginocchio, Phys. Rev. Lett. 78, 436 (1997).

[4] C.E. Vargas and J.G. Hirsch, Phys. Rev. C70, 064320 (2004).

[5] C.L. Wu, Da Hsuan Feng, X.G. Chen, J.Q. Chen, and M.W. Guidry, Phys. Rev. C 36, 1157 (1987).

[6] D. Strottman, Nucl. Phys. A188, 488 (1972)

[7] P. Van Isacker, O. Juillet, and F. Nowacki, Phys. Rev. Lett. 82, 2060(1999).

[8] F. Iachello and A. Arima, The Interacting Boson Model (Cambridge University Press, 1987).

[9] P.J. Elloitt, Proc. Roy. Soc. London A245, 128(1958).

[10] B. Singh, Nucl. Data Sheets, 87, 177 (1999).

[11] R.B. Firestone, Tables of Isotopes (John Willy \& Sons, 1996).

[12] W. G. McKay and J. Patera, Tables of dimensions, indices, and branching rules for representations of simple Lie algebras, Lec- ture Notes in Pure and Applied Mathematics, vol. 69, Marcel Dekker Inc., New York, 1981.

[13] A. Bohr, I Hamomoto, and B.R. Mottelson, Phys. Scr. 26, 267 (1982).

[14] J.N.Ginocchio, Phys. Rep. 414, 165(2005)

[15] A.L. Blokhin, C. Bahri, and J.P. Draayer, Phys. Rev. Lett. 74,4149(1995).

[16] J.N. Ginocchio and A. Leviatan, Phys. Lett. B 425, 1(1998).

[17] R.D. Lawson, Theory of the Nuclear Shell Model (Oxford University Press, 1980).

[18] B. Singh, J.L. Rodriguez, and S.S.M. Wong, Nuclear Data Sheets 84, 487 (1998)

[19] A. Jokinen et al., Eur. Phys. J. A3, 271 (1998).

[20] T. Sekine et al., Nucl. Phys. A 467, 93 (1987).

[21] P. Van Isacker, A. Frank, and J. Dukelsky, Phys. Rev. C 31, 671 (1985). 